\title{
ERRATUM
}

\section{Grapevine Yellows Diseases and Their Phytoplasma Agents}

\section{Biology and Detection}

\section{Marina Dermastia, Assunta Bertaccini, Fiona Constable, and Nataša Mehle}

(C) The Author(s) 2017

M. Dermastia et al., Grapevine Yellows Diseases and Their Phytoplasma

Agents, SpringerBriefs in Agriculture, DOI 10.1007/978-3-319-50648-7

DOI 10.1007/978-3-319-50648-7_5

The original version of this book was inadvertently published with an incorrect affiliation for one of the editors, Nataša Mehle as Department of Agricultural Sciences, Plant Pathology, Alma Mater Studiorum - University of Bologna, Bologna, Italy.

The corrected affiliation has been updated as National Institute of Biology, Ljubljana, Slovenia. 